\title{
$\mathrm{Au}$ cœur de la souffrance
}

Le projet de devenir psychothérapeute spirituel

At the heart of suffering: Plans to become a spiritual psychotherapist

\section{Anne-Cécile Bégot}

\section{(2) OpenEdition}

\section{Journals}

Édition électronique

URL : http://journals.openedition.org/span/707

DOI : 10.4000/span.707

ISSN : 2268-1558

Éditeur

École pratique des hautes études. Sciences humaines

Édition imprimée

Date de publication : 1 juin 2005

Pagination : 121-141

ISSN : 0294-7080

Référence électronique

Anne-Cécile Bégot, «Au cœur de la souffrance», Systèmes de pensée en Afrique noire [En ligne],

17 | 2005, mis en ligne le 05 juin 2013, consulté le 19 avril 2019. URL : http://journals.openedition.org/ span/707; DOI : 10.4000/span.707 


\section{Au cœur de la souffrance Le projet de devenir psychothérapeute spirituel}

Anne-Cécile Bégot

Groupe de sociologie des religions et de

la laicité (GSRL)

EPHE/CNRS

La question du statut professionnel des psychothérapeutes a été au centre du débat politique français à la fin de l'année 2003, avec le projet de loi déposé par le député Bernard Accoyer. L'objectif de ce projet est de réglementer la profession de psychothérapeute, notamment en ne reconnaissant que les seuls diplômés (psychiatres et psychologues) ${ }^{1}$, et en évinçant les autres (psychanalystes et psychothérapeutes) $)^{2}$. La polémique suscitée par ce projet tient, entre autres, au fait qu'il vise à médicaliser la souffrance psychique mais aussi à délégitimer toute une partie de la profession, celle qui exerce sans diplôme reconnu. L'un des arguments utilisés en faveur de ce projet de loi est que n'importe qui peut se déclarer psychothérapeute. Une telle assertion mérite attention, et ce notamment au regard du parcours d'individus rencontrés dans le cadre d'une enquête réalisée pour l'Agence Nationale de Recherches sur le Sida (ANRS).

L'objectif de cette étude était d'étudier la place et le rôle de la spiritualité dans la construction identitaire de personnes touchées par le $\mathrm{VIH} / \mathrm{Sida}^{3}$. Un phénomène relativement singulier est apparu lors de ces rencontres: un certain nombre de ces personnes ont changé de profession, ou manifesté l'intention de le faire, pour devenir psychothérapeute. Bien que ces cas soient peu nombreux (ils concernent quatre personnes), et que l'enquête n'avait pas pour objectif d'étudier leurs parcours professionnels ${ }^{4}$,

\footnotetext{
${ }^{1}$ Voir Loriot, 2004 : 78.

${ }^{2}$ Un amendement séna. torial du 10 juillet 2004 étend la reconnaissance aux psychanalystes. Les psychothérapeutes qui ne sont ni diplômés ni psychanalystes en restent exclus.

${ }^{3}$ Cette étude a été réalisée auprès de trente-huit personnes, sur Paris et sa banlieue, durant deux ans (2000. 2002)

${ }^{4}$ Ce qui explique le manque de données dans certains cas.
}

L'excellence de la souffrance

Systèmes de pensée en Afrique noire, I7, 2005, pp. I2I-I4I 
${ }^{5}$ Ces auteurs ont élaboré une typologie des NMR en trois points : le courant spiritualiste dont fait partie la nébuleuse mystique-ésotérique, le courant conversionniste (ex. les groupes charismatiques) et le courant pré- ou post- milléna. riste (ex. les Enfants de Dieu), voir Davie \& Hervieu-Léger, 1996. ils suscitent l'attention et soulèvent une question à la fois simple et complexe: comment devient-on psychothérapeute? Pour y répondre, on s'attachera à décrire le milieu spirituel dans lequel évoluent ces personnes pour, ensuite, dégager certaines caractéristiques du psychothérapeute spirituel. Chaque parcours étant singulier, on retracera la biographie des quatre personnes tout en mettant l'accent sur les spécificités du métier qu'elles souhaitent exercer.

\section{Des psychothérapeutes mystiques-ésotériques}

Le courant spirituel auquel appartiennent les quatre thérapeutes de l'enquête ANRS est le Nouvel Âge (New Age). Il a émergé à la fin des années 1960 aux Etats-Unis, et au début des années 1970 en Europe. Selon la typologie élaborée par G. Davie et D. Hervieu-Léger (1996), il appartient à un vaste mouvement de renouveau spirituel, qualifié de Nouveaux Mouvements Religieux ou NMR 5 .

Les NMR réunissent des groupes divers et variés et constituent une réponse (en fait plusieurs réponses selon le type de groupe) à une société en crise. Plus précisément, "l'émergence des Nouveaux Mouvements Religieux (NMR) s'inscrit dans le contexte des larges remises en cause des orientations sociales et culturelles des sociétés occidentales qui interviennent à partir de la fin des années 1960 " (Champion, 1995: 13). Inscrits dans le processus de sécularisation (perte de vitesse des religions instituées et prolifération de certaines croyances et pratiques), les NMR ont permis de saisir le vaste mouvement de reconfiguration du religieux au sein des sociétés occidentales. Si les termes NMR traduisent surtout l'absence de consensus entre sociologues sur un autre terme (Champion \& Hourmant, 1999), ils ont néanmoins permis de penser les évolutions du fait religieux au sein des sociétés dites modernes.

Les termes Nouvel Âge ont également suscité des débats (voir Social Compass, 1999) car ils caractérisent une nébuleuse qui ne correspond en rien aux concepts avec lesquels les sociologues des religions travaillaient jusqu'alors (cf. idéaux types de la "Secte " ou de l'"Église »). "On n'y trouve, précise H. Van Hove, ni doctrine ni système de 
croyances intégré. Il [le Nouvel Âge] recouvre des phénomènes aussi divers que le GaiaMind Project sur l'Internet, des best-sellers comme La prophétie des Anges, des formes occidentalisées du Bouddhisme Zen, des cours de massage intuitif, des groupes locaux de méditation pour le salut du monde, des livres d'astrologie s'inspirant de la psychologie de Jung, des thérapeutes découragés qui, dans une étuve indienne, prient pour la guidance, des responsables d'entreprise qui traversent nu-pieds un feu rituel pour célébrer l'anniversaire de leur société " (Van Hove, 1999: 115). L'absence d'institutionnalisation n'empêche pas une large diffusion de cette nébuleuse dans l'ensemble du monde occidental. Les activités du Nouvel Âge se déploient dans des grands centres urbains (Alternatives à Londres, Oibibio à Amsterdam, The Open Center à New York) mais aussi dans des centres locaux (Naple en Floride) voire même des villages (Aups en Provence) (York, 1999 : 175).

Un nombre important d'adeptes du Nouvel Âge sont des « baby boomers " qui, après avoir goûté au mouvement de la contre-culture, ont versé dans la spiritualité (Lewis \& Melton, 1992). Deux périodes doivent être distinguées dans le Nouvel Âge. La première se caractérise par une tendance au messianisme et par l'espérance de temps nouveaux (cf. passage de l'ère du Poisson, marquée par des luttes et tensions, à l'ère du Verseau, temps de paix et d'harmonie). Durant les années 1980, le mouvement évolue, perd sa dimension messianique et devient un vaste milieu où l'on pratique toutes sortes de techniques et disciplines (yoga, méditation, transe...), empruntant à diverses religions (religions orientales mais aussi chamanisme), à l'ésotérisme (astrologie, numérologie), et aux nouvelles thérapies corporelles. Pour différencier ces deux périodes mais aussi pour les caractériser, F. Champion (1995) désigne par « Nouvel Âge » le premier mouvement, et par " Nébuleuse Mystique Ésotérique " le deuxième. La NMÉ a fait l'objet d'une modélisation (construction d'un idéaltype) qui permet de saisir les lignes de force de ce courant spirituel et les différences qui le traversent.

Sept orientations psychospirituelles caractérisent la Nébuleuse Mystique Ésotérique (NMÉ) : la centralité 
${ }^{6}$ Selon Max Weber, deux voies de salut reposent sur l'auto-perfectionnement : la voie éthique où le salut est attaché à un agir conforme à la volonté de Dieu, et la voie mystique où le salut découle de la réalisation d'un certain état d'être, obtenu grâce à un travail de transformation de l'intériorité même du sujet. Dans le cas de la NMÉ ou de la NPM, la voie de salut consiste en la réalisation d'un certain état d'être; « la dimension d'intériorité, de ressenti personnel, $y$ est donc tout à fait centrale »(Champion, 1990 : 27).

${ }^{7}$ Pour V. Rocchi, « le terme "psycho" veut signifier la prédomi. nance des activités thérapeutiques sur les activités religieuses » (Rocchi, 2003 : 177).

8 Les prénoms des personnes ont volon. tairement été changés afin de préserver leur anonymat. donnée à l'expérientiel et son corollaire, « c'est à chacun de trouver sa voie "; la transformation de soi, notamment par des techniques psycho-corporelles ou psycho-ésotériques; le salut, intramondain (bonheur en ce monde), passe par la voie mystique; une vision unifiante du monde et le refus du dualisme des religions abrahamiques; un optimisme certain même s'il reste mesuré; une éthique d'amour; et enfin un jeu du charisme (reconnaissance de l'autorité de certains leaders). Par ailleurs, des lignes de tension existent au sein de la NMÉ : importance accordée ou non aux expériences et situations dites "non ordinaires"; accent mis sur l'expérientiel et/ou l'autoperfectionnement; respect des traditions religieuses et/ou bricolage individuel; orientation psychologique et/ou spirituelle; pratiques magiques et/ou spiritualisme humaniste (Champion, 1995).

À la suite et dans la continuité des travaux de F. Champion, V. Rocchi (2003 : 177) utilise les termes de "nébuleuse psycho-mystique" (NPM). On retiendra cette terminologie car, tout en insistant sur l'élément central de ce courant spirituel, à savoir sa dimension mystique ${ }^{6}$, elle permet aussi d'insister sur le poids important accordé aux aspects thérapeutiques?

Les caractéristiques de la NPM sont présentes, à des degrés divers, dans le parcours des psychothérapeutes rencontrés dans le cadre de l'enquête ANRS. Reste à déterminer quelle formation ces derniers reçoivent pour exercer leur métier. Comment sont-ils formés? Par qui? Comment légitiment-ils un savoir-faire acquis dans une nébuleuse aux frontières floues? Quels sont les critères de validation de ce savoir-faire? Pour répondre à ces questions, on mettra en exergue une thématique forte pour chaque parcours biographique présenté.

\section{La souffrance comme critère de compétence}

Simon $^{8}$, le plus jeune des quatre thérapeutes, a 24 ans quand il apprend sa séropositivité au VIH, en 1991. Il pense avoir été contaminé par l'ami avec qui il vivait à cette époque. D'origine catholique, il a cessé toute pratique reli- 
gieuse relativement jeune mais, durant son adolescence, il a été attiré par des croyances et pratiques ésotériques telles que la réincarnation et l'astrologie. Il dit avoir un « don » de claire-audience et ce, suite à cette voix qu'il a entendu avant de se savoir séropositif.

«Trois mois avant ma séropositivité, raconte-t-il, [...] une voix m'a dit: "n'oublie pas que la maladie n'est pas séparée de l'être”. Jusqu'à présent tout allait bien dans ma vie, enfin c'est ce que je croyais, et je me suis dit pourquoi cette phrase me tombe (dessus). Je l'ai entendue comme si on me parlait à côté, et pourtant j'étais en train de faire les courses avec ma mère, je n'avais pas franchement l'esprit à penser à quoi que ce soit, et sur le moment j'ai compris intuitivement ce que ça voulait dire, ça voulait dire, quelle que soit la maladie [...] ça n'est pas là par hasard. »

À l'annonce de sa séropositivité au VIH, l'engagement spirituel de Simon, jusqu'alors exprimé sur un mode mineur, va devenir beaucoup plus intense. Il fréquente des groupes bouddhistes mais ne poursuit pas dans cette voie, trop normative à son goût. Il se passionne pour un livre écrit par une Américaine, Niro Asistent (1992), qui dit s'être séronégativée, en passant d'un statut sérologique positif au VIH à un statut négatif9. Il poursuit ses investigations auprès d'un maître indien, Osho, qui enseigne la méditation et lui permet de s'inscrire dans une tradition religieuse. Durant cette période, il arrête tout traitement médicamenteux et croit en une possible guérison du Sida.

En 1995, il déclare le Sida avec un cytomégalovirus (CMV) oculaire et voit ses défenses immunitaires s'effondrer. Durant six à huit mois, ses résultats biologiques ne s'améliorent pas et les traitements qu'il prend le font souffrir : "Quand on m'a injecté le produit pour remonter les globules blancs, dit-il, j'avais l'impression d'avoir les os du thorax curés de l'intérieur. "

L'entrée dans la phase Sida bouleverse profondément la vie de Simon. Il réaménage son système de croyances et considère que vivre l'instant présent est plus important que d'obtenir une guérison, ce qui explique son recours aux

${ }^{9}$ La méthode proposée par Niro Asistent repose sur différents aspects : concentration sur soi, se détacher de ses soucis, se concentrer sur ses propres besoins, pratiquer la visualisa. tion créatrice, s'adonner à de l'exercice, s'en. tourer de personnes qui procurent du bien-être (Guérin \& Paillard, 1999 : 64). 
médicaments. Ensuite, il estime avoir changé de caractère : renfermé et peu enclin aux échanges, il dit s'être ouvert aux autres. Enfin, il a effectué un travail sur lui-même : déconstruire certains principes éducatifs et orienter sa vie en fonction de ses désirs et non des normes imposées par la société. Dans cette perspective, il décide d'abandonner son métier de coiffeur pour devenir psychothérapeute. Peu expérimenté, Simon ne vit pas encore de son nouveau métier; les résultats obtenus auprès des quelques clients qu'il a traités le confortent néanmoins dans la voie qu'il a prise.

La formation reçue par Simon est celle qu'il a acquise au gré de stages et rencontres dans la NPM (nébuleuse psycho-mystique). Ainsi, il suivra une thérapie de groupe avec Niro Assistent quand elle s'installera à Paris, en 1998. Il sera également suivi par une psychothérapeute durant un an et demi. L'absence de diplôme ne constitue pas un critère discriminant au sein de ce milieu; au contraire, au regard du parcours de Simon, on se rend compte que la légitimité du psychothérapeute repose sur la maîtrise de techniques psycho-ésotériques et de l'expérience d'une maladie grave.

Utilisant l'astrologie depuis de nombreuses années, Simon s'en servira comme technique de discernement auprès de ses patients. Selon lui, elle l'aide «à comprendre comment fonctionne la personne et surtout, ce qui est important, ce pour quoi elle [la personne] est faite et ce vers quoi elle doit tendre donc ça permet de recentrer plus vite les choses, de moins s'éparpiller, et surtout, d'aller beaucoup plus vite dans l'analyse même si la personne a son temps à elle d'intégration et de transformation. [...] Pour moi le thème astral est une horloge, chaque chose se déclenche à des moments bien précis, les choses doivent se comprendre petit à petit, les unes après les autres. " «De formation réelle? s'interroge Simon, non, je n'en ai pas mais y'a cette forme d'empathie qui est très forte, et ça je ne pense pas que ça s'apprenne; toutes les écoles peuvent vous apprendre des tas de choses merveilleuses mais y'a quand même des choses qu'on ne vous apprend pas c'est la compassion, le non-jugement des autres, l'ouverture à l'autre. " 
Pour Simon, la légitimité de ses compétences thérapeutiques repose sur son expérience de la maladie, et en ce sens, il cherche à se différencier des psychothérapeutes diplômés. "Un psychiatre, explique-t-il, n'a pas eu forcément à traverser une maladie grave ${ }^{10}$ pour se remettre en question et appliquer tous ces principes que je viens d'énumérer [compassion, absence de jugement, ouverture aux autres] et qui sont vachement importants, qui sont essentiels pour pouvoir justement, humainement parlant, s'ouvrir. On me dira que la personne peut l'apprendre au quotidien, en fréquentant ses patients mais je dois dire que les maladies graves, quand même, vous ouvrent des champs d'horizon ${ }^{11}$..." Pour Simon, la maladie ne correspond pas seulement à une altération physiologique de l'être, elle est là pour signifier un mal-être de la personne. "La maladie, dit-il, n'est pas là par hasard [...] pour moi, c'est un signe [la maladie] que la personne, d'une manière ou d'une autre, s'autodétruit. " Trouver la faille, comprendre ce qui pose problème, telle est la démarche de Simon. Il conclut en ces termes : « La maladie n'a fait que me révéler à moi-même. " Ce discours est relativement fréquent chez les adeptes de la NPM, où la maladie est envisagée comme "une épreuve bénéfique qui va permettre à l'homme de dépasser ses problèmes afin d'accéder à un état d'être supérieur appelé "spirituel” " (Rocchi, 2003 : 179).

La maladie est initiatique dans le sens où elle ouvre à la spiritualité (connaissance et ouverture sur le monde supra-empirique) et révèle le "don" de guérison ${ }^{12}$. Ce cas de figure est aussi évoqué par D. Glik (1990) à propos de groupes de guérison dont certains sont issus de la mouvance Nouvel Âge. D'après ses observations, la légitimité du guérisseur (le don qu'on lui reconnaît) repose sur la maladie ou l'épreuve qu'il est parvenu à guérir ou surmonter car cela témoigne de sa foi et/ou de son accès au salut (Glik, 1990 : 153). Dans le cas de Simon, encore relativement isolé, c'est son entourage et plus précisément sa famille qui va le confirmer dans son nouveau statut: «Finalement, dit-il, c'est ma famille qui me reconnaît comme tel [psychothérapeute et astrologue] parce qu'avant j'étais incapable de les aider et

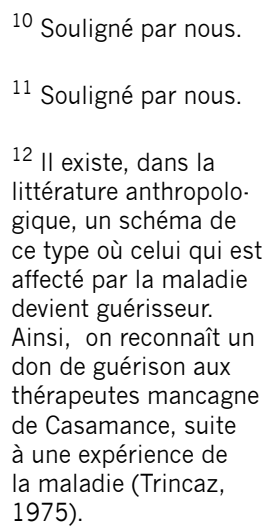


maintenant $[. .$.$] j'arrive à les comprendre au plus profond$ d'eux, ils arrivent à dépasser leurs problèmes, j'arrive à les aider. »

\section{En défiance de la « médecine officielle »}

Xavier, le plus âgé des quatre thérapeutes (une quarantaine d'années), apprend sa séropositivité, en 1986, lorsqu'il décide de se sevrer de l'héroïne. Toxicomane depuis de nombreuses années, il entreprend de suivre le programme des Narcotiques Anonymes (NA) quand, trois mois plus tard, à la suite d'un test de dépistage, il apprend qu'il est séropositif au VIH.

Calqués sur le modèle des Alcooliques Anonymes, les NA ont envisagé un chemin en douze étapes pour parvenir à l'abstinence. L'une d'entre elles consiste à reconnaître l'existence d'une puissance supérieure. Pour Xavier, il s'agira de Dieu. Selon lui, "la drogue était pour moi la recherche d'un Dieu, la transcendance de quelque chose [...]. Je pense que la drogue était une forme de recherche, simplement, je m'étais trompé de Dieu, c'était pas là où il fallait chercher ».

Son engagement au sein des NA est particulièrement intensif. Durant les premières années, il se rend de manière quasi-quotidienne aux réunions prévues par le groupe et parle constamment de Dieu. Il sera le parrain de nombreux filleuls, car le principe du mouvement est qu'une personne abstinente aide (parraine) des néophytes. Il participera aussi à l'organisation et à la structuration du groupe en France. Progressivement, il s'éloignera du mouvement car il s'investit ailleurs (mariage, naissance de son fils, participation à des groupes psycho-spirituels). Il lui reste néanmoins attaché et témoigne occasionnellement, lors de réunions, de ses quinze années d'abstinence.

Jusqu'en 1999, Xavier est dans une démarche thérapeutique alternative et ne ressent pas de symptômes physiques alarmants. À cette date, les évènements se bousculent. Il se fait opérer pour une hernie mais, en sortant de l'hô- 
pital, des maux de tête violents et des vomissements l'alarment. Il retourne à l'hôpital où on lui décèle une méningite triptocoque à laquelle s'ajoutera un CMV oculaire. Durant quatre mois, il est entre la vie et la mort. Ses défenses immunitaires étant quasiment nulles $(2 \mathrm{~T} 4)^{13}$, il maigrit énormément (perte d'une trentaine de kilos), son audition baisse, sa vue est réduite, et il perd une partie de son autonomie. Ce séjour à l'hôpital constitue une étape importante dans le parcours de Xavier, notamment parce qu'il s'est vu se dégrader physiquement mais aussi parce qu'il craignait de mourir dans la souffrance. Régulièrement, l'équipe soignante lui demandait d'évaluer sa douleur sur une échelle de un à dix pour lui administrer, en conséquence, des doses de morphine.

De l'annonce de sa séropositivité (1986) jusqu'en 1999, Xavier n'a donc pas reçu de traitement médicamenteux pour le VIH et s'est orienté vers les médecines dites "parallèles ». Ce choix, il le justifie par l'attitude de ses médecins. "À chaque fois que je voyais des médecins, dit-il, je ressortais et j'avais peur. " Il ajoute que l'un d'entre eux l'a menacé de mort : "Il m'a dit, si vous ne prenez pas l'AZT, vous allez mourir. " Xavier sera conforté dans son choix (ne pas prendre d'AZT) en voyant des personnes séropositives décéder malgré la prise de ce médicament.

Dans le domaine des médecines parallèles, Xavier dit avoir tout essayé : acupuncture, homéopathie, digipuncture $^{14}$, étiopathie ${ }^{15}$, ostéopathie ${ }^{16} \ldots$ et aussi d'y avoir laissé une fortune. L'objectif étant de trouver un équilibre physique, psychologique et spirituel ${ }^{17}$, Xavier s'investira aussi dans des groupes religio-spirituels (zazen, tao sexuel, retraite dans un monastère bouddhiste, intérêt pour l'hindouisme, la Bible...) et réalisera quatre psychothérapies dont une basée sur la sophrologie ${ }^{18}$. Il est difficile de dissocier ces pratiques car avec la NPM on a affaire « à un continuum au sein duquel il est impossible d'établir des frontières nettes entre ce qui relèverait de la nébuleuse psycho-mystiqueésotérique et ce qui relèverait simplement des médecines parallèles, des méthodes de développement personnel, des

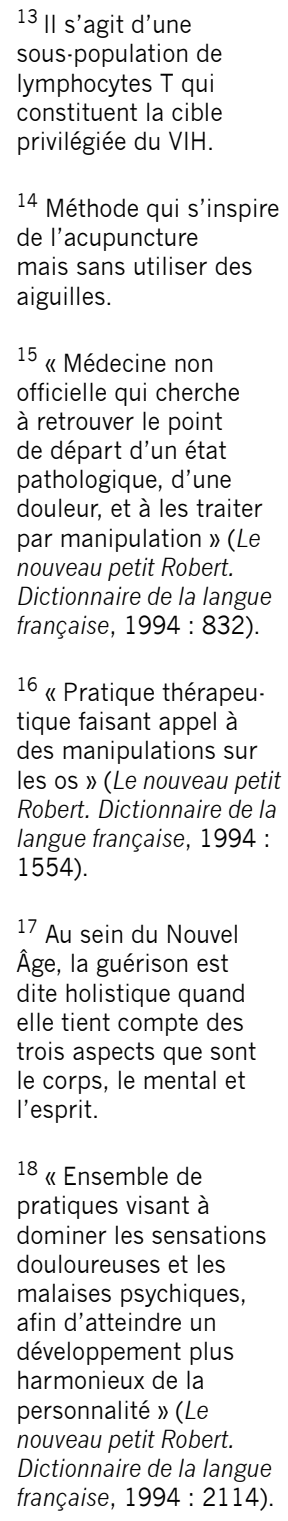

17 Au sein du Nouvel Âge, la guérison est dite holistique quand elle tient compte des trois aspects que sont le corps, le mental et l'esprit.

$$
\begin{aligned}
& 18 \text { « Ensemble de } \\
& \text { pratiques visant à } \\
& \text { dominer les sensations } \\
& \text { douloureuses et les } \\
& \text { malaises psychiques, } \\
& \text { afin d'atteindre un } \\
& \text { développement plus } \\
& \text { harmonieux de la } \\
& \text { personnalité » (Le } \\
& \text { nouveau petit Robert. } \\
& \text { Dictionnaire de la langue } \\
& \text { française, 1994 : 2114). }
\end{aligned}
$$


19 http://

perso.wanadoo.fr/

sidasante/

sousindex.htm psychothérapies en libre service, ou de libres spiritualités " (Champion \& Rocchi, 2000 : 249).

Par ailleurs, et le sociologue Jacques Maître l'a souligné, cette nébuleuse d'hétérodoxie participe au « langage de désir " provoqué par les prouesses scientifiques et médicales. « La modernité, écrit cet auteur, suscite plus d'espoir en la réalisation des désirs qu'elle ne peut en réaliser effectivement, ce qui induit un surplus pour lequel les promesses ne peuvent que venir d'ailleurs" (Maître, 1987 : 363). Ainsi, les thérapies alternatives proposent des remèdes là où la médecine officielle est impuissante et surtout, elles offrent un «supplément d'âme " dont est dépourvu le modèle allopathique.

Entre le milieu des années 1980 et le début des années 1990 diverses alternatives à la médecine officielle vont émerger pour traiter le VIH/Sida. On peut citer la méthode proposée par Guy Claude Burger, l'instinctothérapie, qui consiste à ne manger que des aliments crus, et ce après les avoir humés. Ce théoricien de "l'erreur alimentaire" envisage la maladie (dont le VIH/Sida) comme un moyen naturel de se purifier, d'éliminer les substances nocives accumulées par l'organisme, et considère que l'individu a en soi un "système guérisseur " capable de le guérir (Guérin \& Paillard, 1999 : 47-49) Quant à Marc Griffiths, animateur du site Internet Sidasanté ${ }^{19}$, il s'inscrit dans un courant né aux Etats-Unis, sous l'impulsion d'un rétrovirologue, Peter $\mathrm{H}$. Duesberg. Ce dernier réfute l'équation $\mathrm{VIH}=$ Sida car il a pu observer des cas de Sida chez des patients non séropositifs au HIV. Ce courant considère que la cause des maladies rassemblées sous le nom de Sida est un affaiblissement du «terrain ». La malnutrition, la pollution de l'alimentation, de l'air, de l'eau, les radiations, la chimiothérapie, les transfusions, les excès de stress, les conditions de travail, ... mais aussi des médicaments, dont l'AZT, sont de ces éléments qui fragilisent le «terrain».

Sans appartenir à ce courant alternatif, Xavier a longtemps adhéré à ces idées. On peut replacer un événement important de sa vie dans ce contexte de défiance à l'égard 
de la bio-médecine. Avant que les multithérapies ne soient mises sur le marché (1996), il a tenté, avec sa femme qui est séronégative, de concevoir un enfant. Ils auront pour cela des rapports sexuels non protégés durant une période de six mois, à raison de deux fois par mois. Sa femme, restée séronégative, accouchera d'un petit garçon également séronégatif. Face aux dangers que présentait cette grossesse, Xavier estime qu'ils ont reçu une "grâce ".

Aujourd'hui, Xavier est moins critique à l'égard de l'institution médicale, et ce notamment suite à son séjour hospitalier. Là-bas, dit-il, il a été accueilli par une équipe « extraordinaire » et " humaine ". Quant à son médecin, s'il est un peu railleur et ironique à son égard, il reconnaît qu'il lui a permis de se rétablir. « Mon médecin, dit-il, est très très content de moi parce que je suis une grande réussite pour lui, parce que tous mes résultats sont normaux c'est-à-dire que j'ai une charge virale indétectable donc pour lui c'est une réussite, il est très content, il m'aime beaucoup [rire], il est persuadé de m'avoir sauvé la vie, ce qui est en partie vrai mais pour moi y'a pas que ça. »

Durant treize ans, la position adoptée par Xavier à l'égard de l'institution médicale a été alternative; il s'en est remis aux médecines parallèles et à diverses spiritualités pour se soigner. Il explique son entrée dans la phase Sida par un relâchement de ses pratiques (thérapeutiques et spirituelles). Lors de l'entretien, réalisé en 2001, il adopte une posture complémentaire: il accepte de prendre un traitement médicamenteux (multithérapie) mais considère cette voie comme insuffisante et continue de recourir à la spiritualité (méditation essentiellement) et aux médecines parallèles (acupuncture notamment).

Après s'être rétabli, Xavier concrétise un projet qu'il avait depuis quelque temps: il décide d'exercer comme thérapeute. Il travaille à partir de techniques spécifiques, acquises lors de stages effectués au sein de la NPM. Celle du « dialogue intérieur » lui permet, selon ses termes, d'accéder aux différentes parties (sous-personnalités) qui composent l'individu. 
${ }^{20}$ M. Bowman (1999) note, à la suite d'une étude faite dans la ville de Bath (Grande. Bretagne), haut lieu de la spiritualité Nouvel Âge, que les « guéris. seurs » sont, pour la plupart, des femmes faiblement diplômées.
À la différence de Simon, Xavier ne souhaite pas vivre de ce métier qu'il estime éprouvant. De ce fait, il poursuit son activité d'artisan et consacre une seule journée par semaine à la psychothérapie. Sa crainte est de ne pas être à la hauteur face à ses patients mais son thérapeute le rassure en lui affirmant qu'il a un "don " pour cela. Le bilan hebdomadaire qu'il effectue avec ce dernier atteste, selon lui, de son sérieux et de sa crédibilité.

\section{Des techniques hétérodoxes dans un univers immanent}

Diane, la seule femme sur les quatre thérapeutes ${ }^{20}$, apprend sa séropositivité au VIH en 1989, au moment où son ami déclare la maladie; elle est alors âgée de 27 ans. Quelques années plus tard, en 1993, lorsque son compagnon décède, elle fait une dépression. À l'hôpital où elle est traitée, elle rencontre une infirmière qui lui parle de Dieu. À la différence de Simon et Xavier, Diane a abandonné toute pratique religieuse (catholique) à l'adolescence. Plutôt critique à l'égard des religions, elle ne prête guère d'attention aux propos de cette infirmière jusqu'au jour où elle accepte de rencontrer certains de ses amis qui pratiquent la voyance. D'emblée, Diane est touchée par leur "clairvoyance " car l'un d'entre eux emploie les mêmes termes que ceux de son défunt ami. "Alors là, dit-elle, j'ai eu plein de frissons jusqu'aux oreilles, et j'ai commencé à croire en la vie après la vie. " Ce premier contact est donc décisif.

Non diplômée, Diane a exercé plusieurs types d'emploi. Avant de se savoir séropositive, elle travaillait dans le fret aérien. Licenciée par son entreprise, elle se débrouille pour pouvoir bénéficier d'une pension d'invalidité. Cette situation lui permet de s'investir dans le milieu de la mystique et de l'ésotérisme. Elle achète des livres sur le sujet (spiritualités, médecines parallèles, pratiques ésotériques...), et poursuit son insertion/socialisation dans le milieu en effectuant différents stages : astrologie, tarologie, sophro-relaxation, énergie universelle, chromothérapie, ostéopathie, radiesthésie... Par ailleurs, elle se connecte régulièrement à 
différents sites Internet psycho-ésotériques pour se tenir au courant de l'actualité dans le milieu ${ }^{21}$, et " chate " avec des personnes de même sensibilité qu'elle.

À la différence de Xavier et Simon, Diane s'inscrit d'emblée dans une démarche thérapeutique complémentaire. Ainsi, quand elle déclare le Sida en 1995, pour se soigner, elle combine médecines parallèles et multithérapies.

Deux aspects apparaissent essentiels dans l'engagement spirituel de Diane. En premier lieu, il y a les contacts qu'elle a avec son défunt ami et qui lui permettent, paradoxalement, d'en faire le deuil. Lorsqu'elle consulte des voyants, ces derniers lui assurent que celui-ci est heureux là où il est. Plus tard, elle fera un voyage astral avec sa grand-mère (décédée) qui lui fait traverser la terre entière pour retrouver son ami qu'elle voit comme un «être de lumière ". Cette "expérience " lui permet de tourner une page. «Maintenant, explique-t-elle, je sais que là où il est, il est heureux $[. .$.$] on dit toujours qu'il ne faut pas pleurer les$ morts parce qu'on les retient sur terre; leur âme n'arrive pas à évoluer. Plus on les pleure, moins ils évoluent [...] il faut penser que eux sont bien là où ils sont. "

Le deuxième aspect à prendre en considération est son sevrage de l'héroïne. Toxicomane durant une quinzaine d'années, Diane n'était pas parvenue à arrêter de se droguer, et ce malgré différentes cures de désintoxication. Après avoir pris des produits de substitution, elle s'en est remise à la spiritualité qui lui a permis, selon elle, de s'en sortir.

La place occupée par la spiritualité dans la vie quotidienne de Diane est omniprésente car sa vision du monde est immanente ( $c f$. un monde où ne se distinguent pas forces naturelles et forces surnaturelles). Selon elle, il n'existe pas de hasard dans la vie; tout événement, quel qu'il soit, est voulu par ses «guides spirituels». Au moment de réaliser l'entretien, Diane n'avait plus de voiture; on la lui avait volée. Et d'expliquer : "Je sais pourquoi on m'a volé ma voiture, c'est parce que j'étais trop dispersée, je faisais trop de choses [...] c'était pour me freiner un peu. » De même,

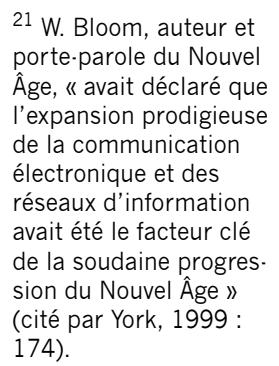
porte-parole du Nouvel Âge, « avait déclaré que l'expansion prodigieuse de la communication électronique et des réseaux d'information avait été le facteur clé de la soudaine progres. sion du Nouvel Âge » (cité par York, 1999 : 174). 
elle considère que les rencontres qu'elle a faites au cours de stages psycho-ésotériques ne sont pas dues au hasard; elles lui ont permis d'évoluer et d'avancer spirituellement. De la même façon, l'expérience proche de la mort (Near Death Experience) qu'elle a vécue après être tombée dans le coma a été, selon elle, un moyen de ne plus craindre la mort, qu'elle envisage, désormais, comme un passage. Son univers spirituel se construit à l'abri de tout contrôle institutionnel et puise dans différentes traditions religieuses et spirituelles.

Diane souhaite devenir naturopathe mais auparavant, elle tient à réaliser un professorat de Chi Qong sur trois ans. Elle a déjà exercé comme thérapeute auprès de son entourage en pratiquant des massages (masso-relaxation) et du reïki. "Quand je fais des séances de reïki, dit-elle, je transmets des énergies, je sens tout mon corps, je sens plein d'énergies dans mes mains, j'aime cette sensation. " Elle raconte qu'elle a dû apprendre à dominer ses émotions et son ressenti car, tels les leveurs de maux (Camus, 1990), elle avait tendance à "prendre le mal " sur elle : "Je sentais la douleur des autres et elle ne partait pas. "Un radiesthésiste va donc lui apprendre à « sentir les énergies des autres mais à ne pas les garder ».

Parmi les multiples techniques hétérodoxes utilisées par les psychothérapeutes spirituels, on peut dégager deux tendances : l'une est plutôt orientée vers la dimension énergétique et la manipulation des corps (cas de Diane), alors que l'autre repose davantage sur un travail d'introspection et d'anamnèse (cas de Xavier).

\section{Parcours, cheminement et constitution d'un réseau}

Thierry, le dernier cas de figure, apprend sa séropositivité en 1986, alors qu'il a vingt-trois ans. Il pense aussi que c'est l'ami avec lequel il vivait qui l'a contaminé. Il décide de terminer ses études et obtient un Diplôme de juristeconseil d'entreprise et un Diplôme d'Études Supérieures Spécialisées (DESS) en droit des affaires et fiscalité. En 
sortant de l'université, il trouve rapidement un emploi et gagne relativement bien sa vie.

Pourtant, au début des années 1990, plusieurs évènements vont le conduire à faire une tentative de suicide. En premier lieu, ses examens biologiques sont mauvais et il présente plusieurs symptômes liés au VIH. Ensuite, il n'apprécie pas la manière dont la société dans laquelle il travaille "gère " le cancer de l'un de ses collègues (absence de soutien, délégation du surplus de travail aux «valides »...). Enfin, sa relation avec son ami se détériore et il rencontre des problèmes financiers.

Après cette tentative de suicide, il part se reposer quelque temps à la montagne. Là, il décide de démissionner de son travail et trouve un poste de directeur juridique dans une entreprise pharmaceutique. À la même période, il vit ce qu'on peut appeler une conversion religieuse ${ }^{22}$, et ce par le biais d'une "guérisseuse ", la sœur d'un ami, qui pratique sur lui une harmonisation ${ }^{23}$. Cette expérience spirituelle va le transformer. Ainsi, il explique qu'après cette harmonisation, il a passé quinze jours de rêve : « Je me levais le matin, j'étais heureux, j'allais bosser, j'étais heureux, je rigolais tout le temps, j'étais super bien. Mon ami me regardait un peu de loin, à la fois en m'encourageant mais aussi en étant un peu dans le doute. Mon psychiatre me regardait de travers. Il y a vraiment quelque chose qui s'est passé. "

De la même façon que Diane, Thierry a abandonné toute pratique religieuse (catholique) à l'âge de l'adolescence, notamment quand il s'est découvert homosexuel. Ce retour vers la spiritualité, ou plutôt la découverte d'un autre univers spirituel, le séduit et l'entraîne dans une spirale qui paraît sans fin. Chercheur en spiritualité, Thierry retrace son histoire en fonction des groupes et thérapeutes qu'il a rencontrés et non en l'inscrivant dans une temporalité précise. Il prend tout d'abord contact avec les groupes de Martin Brofman, l'initiateur de la pratique du corps-miroir (harmonisation). Là, il rencontre un monde parallèle dont il ne soupçonnait pas l'existence : il y est question de visualisations, d'auras ... et il y apprend la technique de l'harmonisation. Il assiste à des conférences, suit de multiples stages,

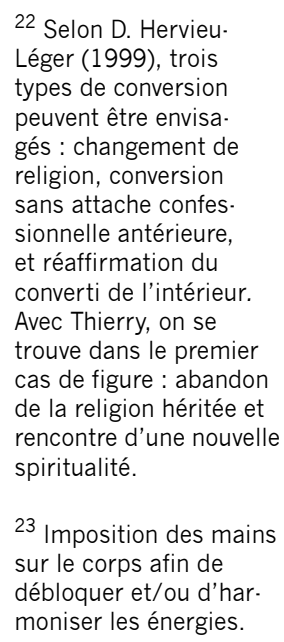
Léger (1999), trois types de conversion peuvent être envisa. gés : changement de religion, conversion sans attache confes. sionnelle antérieure, et réaffirmation du converti de l'intérieur. Avec Thierry, on se trouve dans le premier cas de figure : abandon de la religion héritée et rencontre d'une nouvelle spiritualité.

\footnotetext{
23 Imposition des mains sur le corps afin de débloquer et/ou d'har. moniser les énergies.
} 
${ }^{24}$ Groupes se réclamant de Marthe Robin (1902-1981), une femme ayant reçu les stigmates. se fait soigner par un ostéopathe, pratique le Hatha-Yoga, se forme pour l'accompagnement des mourants puis devient animateur de ces sessions, consulte une psychothérapeute jungienne, suit une formation de chant où l'on applique la technique du dialogue intérieur, veut mettre en place des ateliers d'improvisation sur certains textes bibliques... À la recherche de ses racines religieuses, Thierry va à la rencontre des Rose-Croix d'Or, de Shri Ram Chandra, un groupe religieux d'origine indienne (il ira en Inde rencontrer le gourou de ce groupe, Chariji), des foyers de charité24 ... pour finalement s'arrimer à un groupe orthodoxe syncrétiste. Au cours de l'entretien, il manifeste son souhait de s'engager dans des études de théologie pour devenir prêtre orthodoxe.

Régulièrement, au cours de l'entretien, Thierry fait référence à un monde traversé de forces magiques. Ainsi quand, en 1995, il abandonne tout suivi médical pour le VIH (il ne prend plus de traitement médicamenteux et ne fait plus d'examens biologiques), il justifie cette prise de distance vis-à-vis de l'institution médicale en invoquant son "immunité christique " : "Pour moi, dit-il, c'est la foi qui me porte, c'est le Christ qui est mon immunité, ça peut paraître complètement hallucinant mais ça me réussit plutôt bien. "Et, pour confirmer ses propos, il cite un médecin qu'il a consulté et qui lui aurait tenu ces propos : "Je n'ai quasiment jamais vu ça, vous avez une très légère trace du Sida dans votre ADN, mais y'a rien pour moi, vous êtes quelqu'un qui est en voie de guérison. » Cette croyance dans la toute-puissance de la pensée ou des sentiments est également présente quand il affirme que l'amour peut vaincre la contamination au VIH. Ainsi, il raconte avoir eu des relations sexuelles non protégées pendant un an avec l'un de ses compagnons sans que celui-ci ne soit contaminé car, dit-il, «au fond de moi-même, dans mon cœur, je me dis que l'amour est plus fort que tout mais pas un amour illuminé, un amour ancré, raisonnable qui prend toutes les dimensions de l'être, corps, âme, esprit ». Ce mode de pensée magique existe souvent au sein de la NPM et aussi chez Thierry, personne diplômée du supérieur et bien 
insérée socialement. Par ailleurs, ces croyances magiques peuvent tout à fait cohabiter avec des dispositions éthiques et une vie ascétique.

Dès son immersion dans la NPM, Thierry a l'idée de devenir psychothérapeute. L'un de ses "maîtres " va le conforter dans cette voie : «Il [un thérapeute] n'arrêtait pas de me dire: "Mais c'est incroyable, vous avez une sensibilité, c'est incroyable, j'ai rarement rencontré quelqu'un comme vous, il faut que vous fassiez quelque chose avec, vous ne pouvez pas continuer comme ça, il faut que vous vous formiez, vous avez certainement quelque chose à faire dans l'univers du soin, etc." " Pourtant, rien n'est acquis et, pour constituer sa clientèle, Thierry doit se former et se faire connaître du milieu (Nouvel Âge). La vente de son appartement et ses économies vont lui permettre de patienter en attendant de satisfaire ces exigences.

Avant de véritablement se lancer dans ce métier, Thierry fait une expérience malheureuse comme directeur d'une Maison de la danse. L'échec de cette tentative (licenciement) le conduit à prendre une année sabbatique au cours de laquelle il réfléchit à son avenir professionnel. Déjà à cette période, son réseau relationnel est suffisamment vaste et important pour qu'on lui envoie des patients (deux ou trois par semaine, dit-il). Il les traite avec les techniques qu'il maîtrise, notamment celles du " corps-miroir ". Cette expérience étant, à ses yeux, concluante, il décide de s'engager officiellement comme thérapeute spirituel en ouvrant un cabinet.

À la différence des trois précédents témoignages, Thierry dit vivre de son métier (il a deux cabinets de consultation, l'un à Paris, l'autre en province); il est aussi le seul à faire le lien entre la difficulté d'exercer comme psychothérapeute spirituel et la gestion du phénomène sectaire en France. À ce propos, il dit : « Je suis face à une problématique que je n'ai toujours pas résolue qui est mon inscription sociale, qui est comment m'inscrire dans cette société où il y a une lutte pathologique contre les sectes, que je trouve complètement délirante, où je jongle avec un métier qui n'est pas reconnu socialement. " 
25 Tout psychothéra. peute n'a pas vécu une maladie grave mais, pour ceux qui l'ont vécue, cette expérience contribue, selon eux, à légitimer leur efficacité thérapeutique.
Le cas de Thierry permet de comprendre comment fonctionnent les réseaux au sein de la NPM : ceux-ci s'imposent à l'adepte avant de devenir et/ou d'être envisagés comme une ressource professionnelle. En effet, au départ, l'engagement au sein de la NPM est motivé par une recherche personnelle; les connaissances et techniques acquises au cours des stages et conférences vont servir l'évolution spirituelle de l'adepte. À partir de cette première expérience, celui-ci peut nourrir le projet d'en faire son métier.

\section{Conclusion}

$\mathrm{Au}$ regard des parcours présentés ci-dessus, trois aspects apparaissent comme déterminants dans la formation du psychothérapeute spirituel.

En premier lieu, il y a l'expérience d'une maladie grave, en l'occurrence chronique et létale ${ }^{25}$. Pour les thérapeutes rencontrés dans le cadre de l'enquête ANRS, cette expérience va susciter ou accentuer une recherche de sens qu'ils effectueront dans ce courant spirituel qu'est la " nébuleuse psycho-mystique " (NPM). Là, la maladie s'inscrit dans un modèle étiologique endogène où l'individu est considéré comme responsable de sa maladie, ce qui l'amène à réaliser un travail sur lui-même (transformation de soi), et ce à l'aide de techniques psycho-corporelles ou psychoésotériques. Ce travail constitue une phase initiatique dans le sens où elle le met en contact avec des éléments ou des forces supra-empiriques (Dieu, « intuitions ", « énergies »...) qu'il apprend progressivement à manipuler. L'entrée dans la phase Sida suscite ou accentue ce sentiment d'être possesseur d'un «don " particulier (claire-audience, manipulation d'énergies...), don qui légitime, au regard de ces thérapeutes, le fait de soulager les souffrances de l'autre.

En second lieu, tout psychothérapeute se doit d'avoir, faute d'une formation universitaire et d'un diplôme d'État, une longue expérience au sein de la NPM, expérience constituée de stages, de séminaires, de rencontres... Cette socialisation se traduit par l'acquisition d'un langage spéci- 
fique visant à se conformer aux normes et valeurs de tel ou tel groupe, de techniques thérapeutiques « hétérodoxes », et d'une connaissance d'un milieu qui fonctionne en réseaux. Ainsi, les seuls qui exercent dans un cabinet (Xavier et Thierry) sont dans le milieu depuis une dizaine d'années.

Enfin, en dernier lieu, le capital socio-économique joue un rôle indéniable dans la construction de ce projet. En effet, ouvrir un cabinet nécessite des moyens financiers considérables car la formation du psychothérapeute (cf. toutes les activités pratiquées au sein de la NPM : stages, conférences, thérapies...) est payante et non remboursée. Ce n'est pas un hasard si le seul à avoir fait aboutir son projet (vivre de ce métier) était détenteur d'un capital social et économique conséquent (Thierry), ce qui n'était pas le cas de Simon et de Diane.

À l'origine, rien ne prédisposait Diane, Simon, Xavier et Thierry à (vouloir) devenir psychothérapeute spirituel. Ce projet s'est construit en même temps que leur parcours au sein de la NPM, c'est-à-dire en même temps qu'ils géraient leur séropositivité au VIH. En d'autres termes, pour eux, le métier de thérapeute ne s'est pas improvisé; il s'est acquis au terme d'un long parcours où la souffrance et la maladie ont été envisagées comme critères de compétence.

Je souhaite remercier Françoise Champion pour son travail de relecture et ses remarques stimulantes, ce qui n'empêche que les analyses développées dans ce texte n'engagent que son auteur. 


\section{Références bibliographiques}

Asistent, N.

1992 Comment je me suis guérie du sida et suis redevenue séronégative, Annemasse, Éditions Vivez Soleil.

Bowman, M.

1999 "Healing in the Spiritual Marketplace: Consumers, Courses and Credentialism », Social Compass 46 (2), pp. 181-189.

Camus, D.

1990 Paroles magiques. Secrets de guérison. Les leveurs de maux aujourd'hui, Paris, Imago.

Champion, F.

1990 «La nébuleuse mystique-ésotérique. Orientations psychoreligieuses des courants mystiques et ésotériques contemporains " in F. Champion \& D. Hervieu-Léger (dir.), De l'émotion en religion. Renouveaux et traditions, Paris, Centurion, pp. 17-69.

1995 "Religion et modernité. Nouveaux Mouvements Religieux et nouvelles religiosités mystiques-ésotériques ", Cahiers Français 273, pp. 13-18.

Champion, F. \& L. Hourmant.

1999 "Nouveaux mouvements religieux" et sectes" in F. Champion \& M. Cohen (dir.), Sectes et démocratie, Paris, Seuil, pp. 59-85.

Champion, F. \& V. Rocchi.

2000 "Le soin des âmes et des corps en débat public : l'analyseur psycho-mystique-ésotérique » in P. Bréchon, B. Duriez, J. Ion (dir.), Religion et action dans l'espace public, Paris, L'Harmattan, pp. 241-253.

Davie, G. \& D. Hervieu-Léger.

1996 «Le déferlement spirituel des nouveaux mouvements religieux " in G. Davie \& D. Hervieu-Léger (dir.), Identités religieuses en Europe, Paris, La Découverte, pp. 269-289.

Glik D.C.

1990 "The redefinition of the situation : the social construction of spiritual healing experiences", Sociology of Health and Illness 12 (2), pp. 151-168.

Guérin A. \& B. Paillard.

1999 Médecines parallèles et sida, Paris, CETSAH.

Hervieu-Léger, D.

1999 Le pèlerin et le converti. La religion en mouvement, Paris, Flammarion.

Lewis, J.R. \& J.G. Melton.

1992 Perspectives on the New Age, Albany, State University of New York Press.

Loriot, P.

2004 «Les psys, l'État et les charlatans », Le nouvel observateur, 22/1/2004. 
Maître, J.

1987 "Régulations idéologiques officielles et nébuleuses d'hétérodoxies. À propos des rapports entre religion et santé ", Social Compass 34 (4), pp. $53-364$

Rocchi, V.

2003 «Des nouvelles formes du religieux? Entre quête de bien-être et logique protestataire : le cas des groupes post-Nouvel-Âge en France ", Social Compass 50 (2), pp. 175-189.

Trincaz, J.

1975 «Le pouvoir thérapeutique des Ba-Pena (Casamance). Initiation et pouvoir libérateur ", Psychopathologie africaine 11 (3), pp. 323-362.

Van Hove, H.

1999 "Introduction ", Social Compass 46 (2), pp. 115-119.

York, M.

1999 "Le supermarché religieux : ancrages locaux du Nouvel Âge au sein du réseau mondial », Social Compass 46 (2), pp. 173-179. 